\section{Demenzrisiko erhöht}

Etwa 15\% aller Schlaganfallpatienten leiden an Vorhofflimmern. Forscher der University East Anglia in Großbritannien haben eine Literaturrecherche zum Thema Schlaganfall, Vorhofflimmern und Demenz durchgeführt. Ausgewertet wurden 15 Studien mit insgesamt knapp 47000 Probanden (Durchschnittsalter 72 Jahre). Schlaganfall- patienten mit Vorhofflimmern erkranken demnach 2,4-mal häufiger an Demenz als Schlaganfallpatienten ohne die Rhythmusstörung. Jeder vierte Patient mit Schlaganfall und Vorhofflimmern wurde im Beobachtungszeitraum dement.

Neurology 2011; 76 (10): 914-922 DOI: 10.1212/WNL.0b013e31820f2e38

\title{
PHARMAKOTHERAPIE
}

\section{Beeinflusst die Jahreszeit die Wirksamkeit von Medikamenten?}

Die Verstoffwechselung von Medikamenten wird möglicherweise durch Sonnenlicht beeinflusst, fanden Forscher des Karolinska Institutet in Schweden heraus. Sie werteten ca. 70000 Analysen von Patienten aus, bei denen regelmäßig die Medikamentenspiegel im Blut bestimmt wurden. Es handelte sich dabei um Patienten, die ein Spenderorgan erhalten hatten. Bestimmt wurden die Konzentrationen der Medikamente, die die Organabstoßung verhindern sollten.
Die Blutspiegel verhielten sich umgekehrt proportional zu den Vitamin-D-Spiegeln der Patienten. Vitamin D aktiviert ein Leberenzym (CYP3A4), das den Medikamentenmetabolismus beschleunigt. Möglicherweise müssen also in der Sommerzeit, wenn die Vitamin-D-Spiegel im Organismus am höchsten sind, die Medikamentendosen erhöht werden, wenn man einen bestimmten Spiegel erreichen muss.

Drug Metabolism and Disposition 2011; DOI: 10.1124/ dmd.111.038125

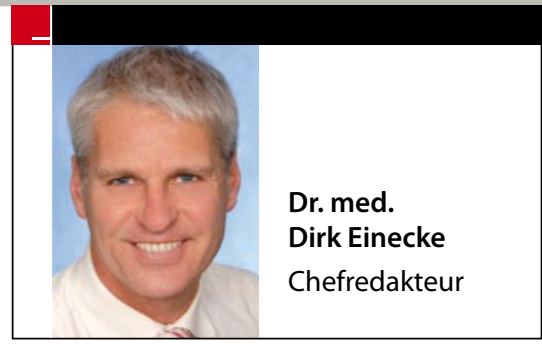

SPORTFANS

\section{Knappe Siege sind lebensgefährlich}

Forscher der North Carolina State University sammelten Daten über schwere Autounfälle nach wichtigen Sportereignissen. Sie wollten wissen, ob Anhänger des Siegeroder des Verliererteams unfallgefährdeter sind und ob es einen Zusammenhang zwischen dem Ausgang des Spiels und der Unfallgefährdung gibt.

Sportfreunde leben dann besonders gefährlich, wenn ihr Team in einem wahren Krimi knapp gewonnen hat. Sie erleiden 133\% mehr schwere Autounfälle als Sportfans, die ein langweiliges Spiel gesehen hatten. Die Freude über den Sieg setzt bei den Betroffenen Testosteron frei und dies verleite sie zu unvorsichtigem Verhalten im Straßenverkehr, vermuten die Forscher.

Pressemitteilung der North Carolina State University

MP3-PLAYER AUF VOLLER LAUTSTÄRKE

\section{Hörschaden zeigt sich im Magnetoenzephalogramm}

Wer häufig über Ohrstöpsel laute Musik hört, schadet seinem Gehör. Der Schaden ist aber mit normalen Hörtests nicht feststellbar. Erst die Magnetoenzephalografie zeigt, ob ein solcher Schaden vorliegt. Das National Institute for Physiological Sciences in Japan und Forscher der Universität Münster wandten die Magnetoenzephalografie auf zwei Gruppen von je 13 Jugendlichen an. Die eine Gruppe hatte regelmäßig laute Musik über Ohrhörer konsumiert, die andere Gruppe nicht. Die Hörfähigkeit war in beiden Gruppen in etwa gleich. Die Fähigkeit, ein bestimmtes Geräusch aus Hintergrundgeräuschen herauszuhören, war aber bei den Musikkonsumenten stark eingeschränkt.

PLoS ONE 2011; 6 (3): e17022 DOI: 10.1371/journal.pone.0017022

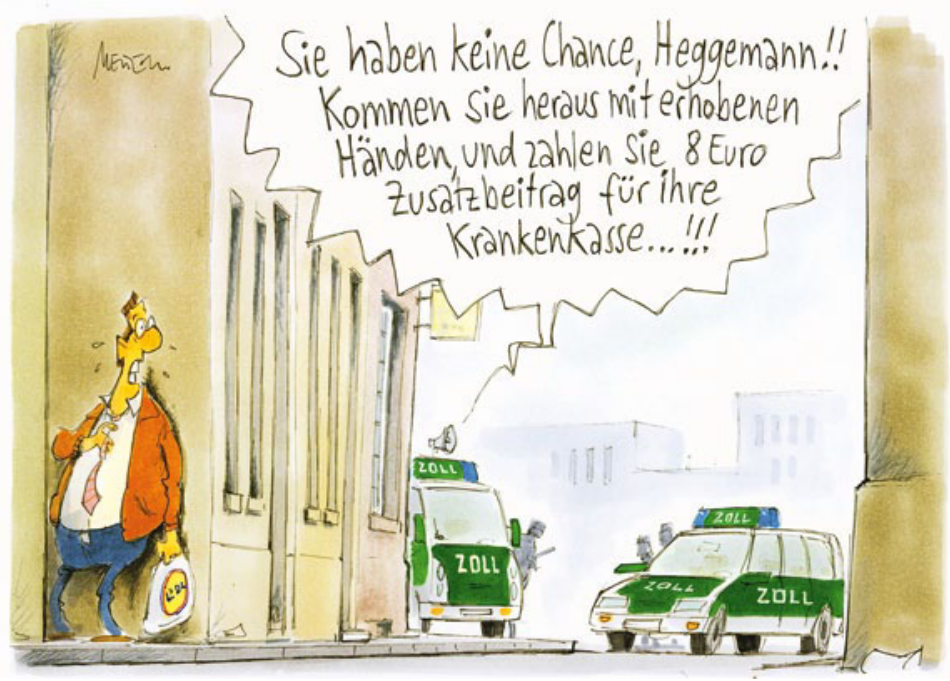

